\title{
Nonlinear Three Photon Process in GaAs and InSb
}

\author{
K Sreelatha ${ }^{1}$, T P Vinu ${ }^{2}$ \\ Department of Physics, NSS Hindu College Changanacherry, Kerala, India
}

\begin{abstract}
Three photon absorption coefficients of GaAs and InSb are studied by considering a non-perturbative approach in which final state is represented by a Volkov wave function. By using perturbative approach the coefficients are calculated by taking a two band model. Here both parabolic and non-parabolic band approximation are used with plane and circularly polarized light. The numerical applications of the three photon absorption coefficients in vicinity of the energy gaps are done for GaAs and InSb.
\end{abstract}

Keywords: Three-Photon absorption, Perturbative, Non-perturbative

\section{Introduction}

The high degree of coherence of laser radiations had made it possible to have extremely high spacial concentration of light power. Due to coherence the electric field associated with the light beam become comparable to the inter atomic electric field. At such high electric fields, the relationship between the electric polarization $\mathrm{P}$ and the field strength $\mathrm{E}$ ceases to be linear and some nonlinear effects come to the fore. In physics and other sciences, a nonlinear system, in contrast to a linear system, is a system which does not satisfy the super position principle.

The existence, character and magnitude of non-linearity provide more information about the structure of matter. The nonlinear terms in the interaction of light with atoms gives rise to a variety of non-resonant phenomenon so called multi-photon absorption. Usually the multi-photon processes are obtained by using the high power pulsed laser such as ruby laser, Nd:YAG laser, Nd:Glass laser etc. This study is a theoretical calculation of three photon absorption coefficients of two semiconducting material, using Volkov type wave function, by a perturbative and non-perturbative approach.

\section{Literature Survey}

Two-photon absorption means is the simultaneous absorption of two photons of same or various frequencies to excite or raise a molecule from one state to a higher energy electronic state. The base state are usually the ground state. The energy difference between the involved lower and upper states of the molecule will be equal to the sum of the energies of the two photons. This is not a linear process but a nonlinear optical process.

Three-photon absorption, hereinafter 3PA, is a nonlinear absorption process, in which three photons are absorbed simultaneously and a molecule is boosted or excited from a lower energy level, normally ground level to a higher energy level.

The elementary process of 3PA can be explained in three steps

1) The first step, one photon is absorbed while the molecule leaves its initial state to be exited to an intermediate state

2) Secondly, the molecule absorbs second photon and make a transition to another intermediate state.

3) Finally, third photon is absorbed and the excited molecule reaches its final excited state

\section{Methodology}

In this study we used perturbative and non-perturbative treatments for calculating the 3PA coefficient.

\section{Perturbative approach}

For the three photon absorption, we have,

$\alpha^{(3)}=2^{\frac{3}{2}} 3^{2} B m_{c v}^{2}|M|^{2} \frac{\left(3 \hbar \omega-E_{g}\right)^{\frac{5}{2}}}{(\hbar \omega)^{7}}$

for parabolic bands and

$$
\alpha^{(3)}=\frac{3^{3} B\left(m_{c v}\right)^{2}|M|^{2}}{2(\hbar \omega)^{6}}\left\{\left(\frac{3 \hbar \omega}{E_{g}}\right)^{2}-1\right\}^{\frac{5}{2}}
$$

for non- parabolic bands

Here we consider only initial valence band and final conduction band. Two of the dipole matrix elements are forbidden where the transition mechanism is of an allowedforbidden-forbidden-type.

\section{Non-perturbative approach}

Iqbal and Hassan have calculated the interband multiphoton absorption coefficient using a non-perturbative approach. due to a modified Keldysh theory based on the Volkov type final state wave functions and the two-band model. From this approach, one can obtain the following forms for the 3PA coefficients:

$\alpha^{(3)}=\sqrt{2} B^{\prime}|M|^{2} \frac{\left(3 \hbar \omega-\dot{E}_{g}\right)^{\frac{5}{2}}}{(\hbar \omega)^{7}}$

for parabolic bands and plane-polarized photon,

$\alpha^{(3)}=\frac{\sqrt{2}}{6} B|M|^{2} \frac{\left(3 \hbar \omega-\dot{E}_{g}\right)^{\frac{5}{2}}}{(\hbar \omega)^{7}}$

for parabolic bands and circular- polarized photon,

$\alpha^{(3)}=\frac{\sqrt{2}}{2^{3}} B^{\prime} E_{g}^{\frac{3}{2}}|M|^{2} \frac{(3 \hbar \omega-\beta)}{(\hbar \omega)^{7}}\left[\left(\frac{3 \hbar \omega-\beta}{E_{g}}\right)^{2}-1\right]^{\frac{5}{2}}$

for non- parabolic bands and plane-polarized photon, and 


\section{International Journal of Science and Research (IJSR) \\ ISSN (Online): 2319-7064}

Index Copernicus Value (2013): 6.14 | Impact Factor (2014): 5.611

$\alpha^{(3)}=\frac{\sqrt{2}}{2^{3} \times 6} B^{\prime} E_{g}^{\frac{3}{2}}|M|^{2} \frac{(3 \hbar \omega-\beta)}{(\hbar \omega)^{7}}\left[\left(\frac{3 \hbar \omega-\beta}{E_{g}}\right)^{2}-1\right]^{\frac{p}{2}}$

for non-parabolic bands and circular polarized photon.
Where the parameters used in the above mentioned formulas, are general conventions used in literature.

Table 1

\begin{tabular}{|c|c|c|c|c|c|c|c|}
\hline \multicolumn{8}{|c|}{ Three photon absorption coefficient of materials } \\
\hline \multirow[t]{3}{*}{ Materials } & \multirow[t]{3}{*}{$\hbar \omega(\mathrm{eV})$} & \multirow{2}{*}{\multicolumn{2}{|c|}{\begin{tabular}{|l|} 
Perturbative approach \\
Two-band $\left(\mathrm{cm}^{-1}\right)$
\end{tabular}}} & \multicolumn{4}{|c|}{ Non-perturbative approach } \\
\hline & & & & \multicolumn{2}{|c|}{ parabolic $\left(\mathrm{cm}^{-1}\right)$} & \multicolumn{2}{|c|}{ Non-parabolic $\left(\mathrm{cm}^{-1}\right)$} \\
\hline & & Parabolic & Non-parabolic & Plane & Circular & Plane & Circular \\
\hline GaAs & 2.6 & $2.688 \times 10^{-11}$ & $7.862 \times 10^{-11}$ & $6.9025 \times 10^{-14}$ & $1.1505 \times 10^{-14}$ & $4.9275 \times 10^{-12}$ & $2.3220 \times 10^{-12}$ \\
\hline InSb & 2.6 & $6.1797 \times 10^{-14}$ & $1.252 \times 10^{-14}$ & $1.4461 \times 10^{-15}$ & $2.4102 \times 10^{-16}$ & $4.5496 \times 10^{-11}$ & $2.1450 \times 10^{-11}$ \\
\hline
\end{tabular}

\section{Conclusion}

The Absorption coefficient which determines how far into a material light of a particular wave length can penetrate before it is absorbed. By the study of multi-photon absorption coefficient we can give detailed information about the energy band structures not readily attainable by using linear techniques

We have performed a theoretical calculation of 3 PA coefficients using perturbative and non-perturbative approaches using parabolic and non-parabolic band approximations. The calculations are done with both plane polarized and circularly polarized light. The numerical applications of the three photon absorption coefficients in vicinity of the energy gaps are done for GaAs and InSb are given in table 1 . The band parameters and effective masses are taken from literature.

\section{Future Scope}

Multi photon absorption process have been successfully used to produce population inversion in semiconducting laser materials. Here we studied only about GaAs and InSb. Future work should can on "understanding the optical properties" of semiconducting materials like InSb or GaAs and combinations of other semiconducting materials.

\section{References}

[1] F Bassani and A R Hassan, Nuovo Cimento, 1972,7B, 313.

[2] A R Hassan, Physics. Stat.Solidi (b), 1990, 160, 185.

[3] M Zafar Iqbal and A R Hassan, Solid State Communication, 1989, 479, 1970.

[4] L V Keldysh Sov. Physics, JETP, 1965,20, 1307.

[5] H D Johns and HR Reiss, Phys. Rev 1977,B16, 2466.

[6] F. Adduci, IM Catalano, Phy. Rev, 1977,B15, 926.

[7] M Claudiu, Cirloganu et al. Optics express 2011, 19, 22951

\section{Author Profile}

Dr. K Sreelatha, Asst Professor, Department of Physics, NSS Hindu College Changanacherry, (under Mahatma Gandhi University, Kottayam Kerala).

Dr. TP Vinu, Asst Professor, Department of Physics, NSS Hindu College Changanacherry, (under Mahatma Gandhi University, Kottayam Kerala). 